\title{
Processamento de estruturas tridimensionais de Medicina Nuclear na modalidade PET
}

\author{
Edward Flórez Pacheco*, Sérgio Shiguemi Furuie
}

Resumo A Medicina Nuclear, como especialidade de obtenção de imagens médicas é um dos principais procedimentos utilizados hoje nos centros de saúde, tendo como grande vantagem a capacidade de analisar o comportamento metabólico do paciente. Este projeto está baseado em imagens médicas obtidas através da modalidade PET (Positron Emission Tomography). Para isso, foi desenvolvida uma estrutura de processamento de imagens tridimensionais PET, constituída por etapas sucessivas que se iniciam com a obtenção das imagens padrões (gold standard), sendo utilizados para este fim volumes simulados do Ventrículo Esquerdo do Coração criadas como parte do projeto, assim como phantoms gerados com o software NCAT-4D. A seguir, nos volumes simulados é introduzido ruído Poisson que é o ruído característico das imagens PET. Na sequência é executada uma etapa de pré-processamento, utilizando alguns filtros 3D tais como o filtro da mediana, o filtro da Gaussiana ponderada e o filtro Anscombe/Wiener. Posteriormente é aplicada a etapa de segmentação, processo baseado na teoria de Conectividade Fuzzy sendo implementadas quatro diferentes abordagens 3D: Algoritmo Genérico, LIFO, kTetaFOEMS e Pesos Dinâmicos. Finalmente, um procedimento de avaliação conformado por três parâmetros (Verdadeiro Positivo, Falso Positivo e Máxima Distância) foi utilizado para mensurar o nível de eficiência e precisão do processo. Constatou-se que o par Filtro - Segmentador constituído pelo filtro Anscombe/Wiener junto com o segmentador Fuzzy baseado em Pesos Dinâmicos proporcionou os melhores resultados, com taxas de VP e FP na ordem de 98,49 $\pm 0,27 \%$ e 2,19 $\pm 0,19 \%$, respectivamente, para o caso do volume do Ventrículo Esquerdo simulado. Com o conjunto de escolhas feitas ao longo da estrutura de processamento, encerrou-se o projeto analisando um número reduzido de volumes pertencentes a um exame PET real, obtendo-se a quantificação dos volumes.

Palavras-chave Medicina nuclear, Tomografia por Emissão de Pósitrons (PET), Processamento de imagens médicas 3D, Segmentação de imagens 3D, Quantificação dos volumes.

\section{Processing of three-dimensional structures of Nuclear Medicine in PET modality}

Abstract The Nuclear medicine, as a specialty to obtain medical images is very important, and it has became one of
the main procedures utilized in Health Care Centers to analyze the metabolic behavior of the patient. This
project was based on medical images obtained by the PET modality (Positron Emission Tomography). Thus,
we developed a framework for processing Nuclear Medicine three-dimensional images of the PET modality,
which is composed of consecutive steps that start with the generation of standard images (gold standard) by
using simulated images of the Left Ventricular Heart, such as phantoms obtained from the NCAT-4D software.
Then, Poisson quantum noise was introduced into the whole volume to simulate the characteristic noises in PET
images. Subsequently, the pre-processing step was executed by using specific 3D filters, such as the median
filter, the weighted Gaussian filter, and the Anscombe/Wiener filter. Then the segmentation process, which is
based on the Fuzzy Connectedness theory, was implemented. For that purpose four different $3 D$ approaches
were implemented: Generic, LIFO, kTetaFOEMS, and Dynamic Weight algorithm. Finally, an assessment
procedure was used as a measurement tool to quantify three parameters (True Positive, False Positive and
Maximum Distance) that determined the level of efficiency and precision of our process. It was found that
the pair filter - segmenter formed by the Anscombe/Wiener filter together with the Fuzzy segmenter based
on Dynamic Weights provided the best results, with VP and FP rates of $98.49 \pm 0.27 \%$ and $2.19 \pm 0.19 \%$,
respectively, for the simulation of the Left Ventricular volume. Along with the set of choices made during the
processing structure, the project was finished with the analysis of a small number of volumes that belonged
to a real PET test, thus the quantification of the volumes was obtained.

Keywords Nuclear medicine, Positron Emission Tomography (PET), 3D medical image processing, Segmentation of $3 D$ images, Volume quantification.

*e-mail: edward.florez@usp.br

Recebido: 17/08/2012 / Aceito: 14/12/2012 


\section{Introdução}

$\mathrm{Na}$ atualidade existem várias modalidades que tornam possível a obtenção de imagens médicas, entendendo-se por imagem médica o conjunto de técnicas, métodos ou procedimentos através dos quais é possível obter imagens de um organismo vivo, ou parte dele (Bushberg et al., 2002). As quatro principais modalidades de obtenção de imagens médicas utilizadas hoje na medicina são: Ultrassom, Ressonância Magnética, Raios X/Tomografia Computadorizada e Medicina Nuclear. Todas elas têm como precursor os Raios X, o qual existe há um século de antiguidade (Caetano, 2002). Cada uma destas modalidades possui um princípio físico diferenciado que lhe permite obter diferentes tipos de informações dos pacientes. Assim mesmo, cada modalidade apresenta vantagens e limitações, o qual faz que cada uma delas seja adequada para aplicações específicas.

Atualmente, é crescente a aceitação e utilização da Medicina Nuclear onde encontramos a Tomografia por emissão de pósitrons (PET, acrônimo do inglês Positron Emission Tomography). Com princípios claramente diferenciados daquelas ferramentas não invasivas que os especialistas tinham para obter imagens, surgiu o PET como uma inovadora modalidade de imagem médica, através da qual foi possível obter imagens funcionais do interior do organismo. O PET é uma modalidade de imagem médica que outorga a possibilidade de reconhecer alterações num órgão, função e/ou estrutura específica de seres humanos e/ou de animais, com peculiar sensibilidade e especificidade na detecção de doenças. Consegue também demonstrar precocemente desordens, transtornos ou alterações bioquímicas, mesmo onde não existe (ainda) uma anormalidade estrutural evidente, permitindo um diagnóstico mais precoce e com maior possibilidade de acerto (De Almeida et al., 2002; Robilotta, 2006).

O PET possibilitou à Medicina Nuclear observar a distribuição espacial e temporal de marcadores fisiológicos e funcionais, permitindo avaliação quantitativa e não invasiva de processos fisiológicos. Estes marcadores podem indicar desde o funcionamento anômalo de órgãos até a bioquímica no metabolismo celular. Sua principal aplicação consiste na investigação de tumores e metástases, que naturalmente têm aumentado o consumo de glicose, devido ao maior nível metabólico. Se o estudo é realizado em vários intervalos sucessivos de tempo, obtém-se uma distribuição temporal de imagens do marcador, possibilitando estudos dinâmicos (Tinois, 2005).

O futuro do PET é auspicioso, ajudado com o desenvolvimento de novos radiofármacos e materiais detectores, técnicas de reconstrução mais eficientes, entre outros avanços. No entanto, ainda há inúmeros mecanismos fisiológicos que são desconhecidos ou pouco esclarecidos, e o PET é uma importantíssima ferramenta para investigar hipóteses e modelos de fenômenos biológicos, inclusive na efetividade das diferentes terapias. Neste processo, há uma carência muito grande em relação a técnicas e ferramentas computacionais que permitam avaliar e estudar a dinâmica de processos a partir de imagens tridimensionais de PET.

O projeto terá como foco o estudo do Ventrículo Esquerdo do Coração, no entanto, como ferramenta de aplicação não ficará limitada a esta região. A escolha desta região, deve-se ao fato de representar uma área associada a um grave problema de saúde pública, refletido estatisticamente num número considerável de pacientes com CAD (do inglês Cardiac Artery Disease) e insuficiência cardíaca (Pedone et al., 2004). A função do Ventrículo Esquerdo (VE) é um importante indicador para o diagnóstico e prognóstico dos pacientes com CAD (Johnson et al., 1991; Shaw et al., 1998).

Tendo em consideração que as imagens PET e de Medicina Nuclear apresentam uma pobre relação sinal-ruído, originado principalmente pela baixa contagem de fótons e pelo ruído Poisson, característico nesse tipo de imagens (King et al., 1983), o principal objetivo do projeto é a pesquisa e desenvolvimento de métodos para a análise de processos fisiológicos baseado em PET. A relevância deste trabalho consiste no desenvolvimento de métodos computacionais de processamento que permitirão avaliar estruturas de imagens 3D de Medicina Nuclear na modalidade PET, possibilitando a analise de volumes de interesse ao longo do tempo.

\section{Materiais e Métodos}

\section{Processos físicos na técnica PET}

Com princípios claramente diferenciados daquelas ferramentas não invasivas que os especialistas tinham para obter imagens, surgiu a Medicina Nuclear como uma inovadora modalidade de imagem médica, através da qual foi possível obter imagens funcionais do interior do organismo. A Medicina Nuclear é uma especialidade médica que envolve o uso de materiais radioativos denominados radiofármacos no estudo, diagnóstico, terapia e no tratamento de várias condições clínicas e doenças (De Almeida et al., 2002).

Existem dois tipos de exames tomográficos utilizando o princípio de Medicina Nuclear, diferenciando-se uma da outra pela ocorrência de liberação de radiação gama: Quando existe emissão de pósitrons os quais interagem com os elétrons 
e geram dois fótons simultâneos e em direções praticamente opostas, a técnica é chamada de PET (Positron Emission Tomography). Por outro lado, quando a emissão não é pósitron, mas sim fóton, como resultado do decaimento do radiofármaco, a técnica é chamada de SPECT (Single Photon Emission Computed Tomography).

A imagem obtida com o PET inicia-se com a administração ao paciente (por via endovenosa) de uma substância metabolicamente ativa, que carrega um isótopo emissor de pósitron, funcionando como um traçador (Oliveira et al., 2006). Depois de alguns minutos, o isótopo vai se alojar na área do corpo onde a molécula tem afinidade. O núcleo radioativo instável decai a um estado mais estável pela emissão de elétrons carregados positivamente (isto é, pósitron). $\mathrm{O}$ pósitron ejetado combina-se com um elétron e quase instantaneamente essas duas partículas sofrem um processo de aniquilação por terem cargas iguais e opostas, emitindo sua energia de repouso sob a forma de dois fótons de alta energia (fótons de $511 \mathrm{keV}$ ). Essa radiação gama emerge do corpo em direções praticamente opostas e são posteriormente detectados por uma combinação de cristais de cintilação que converte os fótons de aniquilação em luz visível, sendo que eventualmente ocorre um desvio dos fótons emitidos (fenômeno conhecido como dispersão ou scattering) que influirá na qualidade da imagem. $\mathrm{Na}$ sequência, através de um tubo fotomultiplicador, é convertida a luz visível num sinal eletrônico originando finalmente a imagem médica (Wagner Junior, 1998).

Depois da detecção de um grande número de eventos produzidos pela aniquilação de pósitrons e elétrons, a distribuição do traçador é computacionalmente calculada a partir dos dados de projeção registrados. Por meio de um computador é processada essa distribuição, e através de procedimentos de reconstrução são obtidas as respectivas imagens transversas, coronais e sagitais (National..., 1996; Wagner Junior, 1998).

Projeções unidimensionais obtidas em diferentes ângulos tornam possível reconstruir uma imagem bidimensional, assim como também se pode fazer reconstruções tridimensionais a partir de projeções bidimensionais (Fabey, 1996; Peregrino et al., 2004). As imagens resultantes correspondem a medidas da distribuição do isótopo, podendo ser usadas tanto para avaliar, qualitativamente, o local de acúmulos não usuais do traçador (por exemplo, em tumores), quanto para medir, quantitativamente, a captação do traçador, para um diagnóstico mais profundo ou a localização de uma doença (Adams et al., 1999).

No processo de geração das imagens PET, têm envolvidas algumas características específicas desta modalidade tais como: alcance do pósitron, não colinearidade, dispersão e atenuação, eletrônica, radioquímica que são detalhadas em Flórez Pacheco (2012).

As imagens obtidas através da modalidade PET vêm sendo utilizadas para avaliar uma variedade de doenças, com base em seus processos funcionais, metabólicos, bioquímicos e fisiológicos. Basicamente suas principais aplicações clínicas ficam concentradas em três grandes áreas: oncologia, cardiologia e desordens neuropsiquiátricas.

\section{Sequência de processamento de imagens}

Processar uma imagem consiste na sua manipulação através de técnicas que objetivam a extração da informação nela contida (Gonzalez e Woods, 2008), visando uma melhoria de informação visual para interpretação humana ou para alguma aplicação específica.

Entre o conjunto de tarefas que conformam o processamento das imagens, existe uma inter-relação estreita entre cada um dos passos envolvidos neste processo, já que cada uma destas etapas contribui a que as imagens que estão sendo analisadas se tornem adequadas, ou pelo menos com melhores características para o sucesso do processo ou etapa posterior.

A estrutura de processamento proposta neste trabalho está constituída pelas seguintes partes: (i) Aquisição e/ou simulação de imagens tridimensionais de Medicina Nuclear na modalidade PET; (ii) Adição de ruído tipo Poisson nos volumes simulados; (iii) Pre-processamento: filtragem de imagens tridimensionais; (iv) Segmentação de estruturas de interesse nas imagens tridimensionais; (v) Avaliação dos resultados; e (vi) Quantificação dos volumes.

Para o desenvolvimento dos algoritmos que fazem parte da estrutura de processamento, foi utilizado um computador Intel Core 2 Duo, $2.4 \mathrm{GHz}$, com 3 GB de memória RAM e 120 GB de espaço em disco rígido. Todos os algoritmos aplicados são exibidos em Flórez Pacheco (2012). Além disso, é importante indicar que as plataformas escolhidas para o desenvolvimento dos algoritmos deste projeto foram basicamente o Java/Eclipse (http://www.eclipse.org/) e o ImageJ (http://rsbweb.nih.gov/ij/), complementando algumas tarefas específicas com alguns códigos feitos em MatLab.

\section{Aquisição/simulação das imagens 3D}

A aquisição de uma imagem está ligada diretamente ao processo de captura da imagem, usando um método específico (Gonzalez e Woods, 2008). Obviamente é fundamental que as imagens capturadas sejam de alta qualidade (Russ, 1998), de tal forma que a informação contida nela seja fácil e claramente interpretada. 
Imagens simuladas da região requerida foram utilizadas. Por um lado, simulações de volumes fisiológicos obtidos através da ferramenta NCAT-4D (Nurbs-based Cardiac-Torso), proporcionados pelo Serviço de Informática do Instituto do Coração (InCor) do Hospital das Clínicas da Faculdade de Medicina da Universidade de São Paulo (HC-FMUSP). Além disso, imagens 3D próprias geradas através de MatLab, serviram como volumes de entrada na sequência de processamento.

Estes phantoms cumprem um papel cada vez mais preponderante nas pesquisas com imagens médicas, já que têm a capacidade de simular um número ilimitado de anatomias conhecidas dos pacientes, oferecendo meios práticos para avaliar quantitativamente, comparar e melhorar técnicas e dispositivos de imagens médicas (Segars e Tsui, 2009). A seguir apresentamos algumas das mais importantes características e detalhes dos phantoms utilizados neste trabalho:

\section{NCAT (Nurbs-based Cardiac-Torso)}

O NCAT-4D foi desenvolvido para a pesquisa de imagens de medicina nuclear, especificamente para tomografia computadorizada por emissão de fóton único (SPECT) e tomografia por emissão de pósitrons (PET) (Segars e Tsui, 2009). Esta ferramenta fornece um modelo realista da anatomia humana, envolvendo movimentos cardíacos e respiratórios, fazendo possível a simulação de uma série de phantoms 3D durante um determinado período de tempo. Tanto o período de tempo, o número de phantoms de saída, quanto o tipo de movimentação requerido são definidos pelo usuário através de parâmetros determinados.

Na Figura 1 é mostrada a simulação do Ventrículo Esquerdo do Coração junto com a estrutura da caixa torácica, este phantom está formado por um grupo de 16 frames sendo que cada um dos volumes está constituído por um conjunto de 20 fatias. Em Flórez Pacheco (2012) são apresentados os principais parâmetros de construção deste phantom, que será objeto de estudo no projeto.

\section{Imagens 3D do Ventrículo Esquerdo Simulado}

Sendo o foco de nosso estudo o ventrículo esquerdo do coração, foi simulada esta cavidade tomando em consideração aspectos como dimensões e a espessura da sua parede, mencionadas na literatura (Fifer e Grossman, 2000; Wikipedia, 2011).

Em Flórez Pacheco (2012) estão detalhados os procedimentos, os parâmetros e os algoritmos que permitiram gerar os 30 volumes (cada volume composto por 128 fatias) posteriormente testados. $\mathrm{Na}$ Figura 2, apresenta-se um modelo simples do volume da cavidade do Ventrículo Esquerdo simulado.

Além disso, utilizou-se também um conjunto de volumes pertencentes a um exame real PET dinâmico cardíaco, adquirida no Instituto do Coração do Hospital das Clínicas da Faculdade de Medicina da Universidade de São Paulo (InCor-HCFMUSP), protocolado pela Comissão de Ética para Análise de
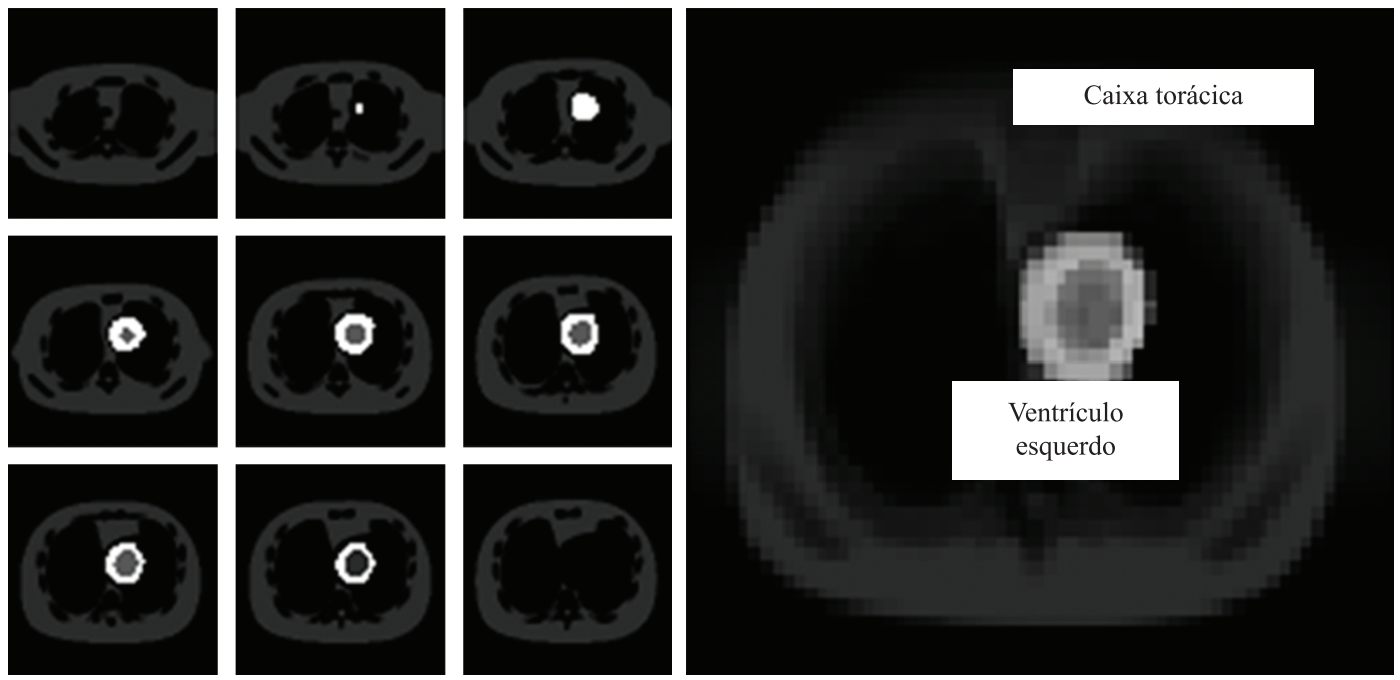

Figura 1. Phantom simulado com o uso do NCAT-4D. (a) Fatias 2D das projeções do ventrículo esquerdo dentro da caixa torácica; (b) Volume gerado com ajuda das ferramentas do ImageJ.

Figure 1. Simulated phantom using the NCAT-4D. (a) 2 D slices of the projections of the left ventricle in the chest cavit; (b) Volume generated with the aid of ImageJ tools. 
Projetos de Pesquisa do HCFMUSP (Cappesq), com número 0630/07. O exame PET real foi realizado num paciente de sexo masculino, conformado por um conjunto de 36 volumes, adquiridas em períodos de 300 segundos cada. A Figura 3 mostra por um lado a sequência de algumas das fatias do miocárdio estudado e por outro o volume 3D reconstruído. Em Flórez Pacheco (2012) são apresentados os dados mais relevantes deste exame PET real, assim como uma descrição detalhada dos passos para o processo de reconstrução deste grupo de volumes PET reais.

\section{Adição do ruído quântico tipo Poisson}

Os volumes simulados, tanto do Ventrículo Esquerdo do Coração desenvolvido em MatLab quanto daqueles obtidos através do NCAT-4D, foram adquiridas inicialmente sem nenhum tipo de ruído. Segundo a literatura, as imagens PET são corrompidas pelo ruído quântico que é dependente do sinal e pode ser modelado por uma distribuição estatística de Poisson (Bethea e Duran, 1995; Romualdo, 2009). Por se tratar de um ruído aleatório, a modelagem do ruído quântico é baseada apenas em ferramentas estatísticas.

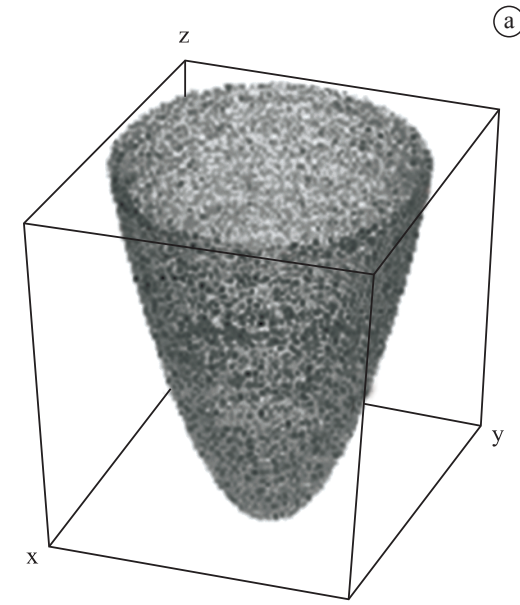

(a)

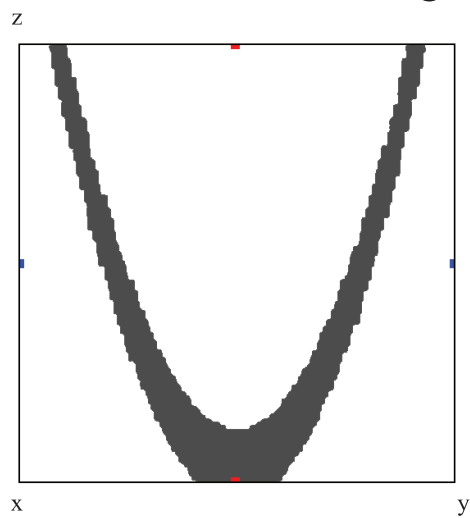

Figura 2. Phantom tridimensional do Ventrículo Esquerdo (mudança do contraste em relação ao phantom original com o propósito de aprimoramento da visualização). (a) Volume gerado por meio de ferramentas de reconstrução do ImageJ; (b) Projeção frontal nos eixos y-z. Figure 2. Tri-dimensional phantom of the Left Ventricle (alteration of the contrast of the original phantom in order to improve it visualization). (a) Volume generated by means of ImageJ reconstruction tools; (b) Frontal projection at y-z axes.

(a)
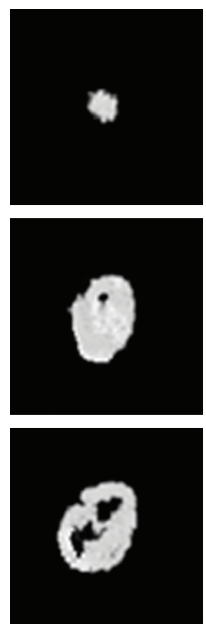
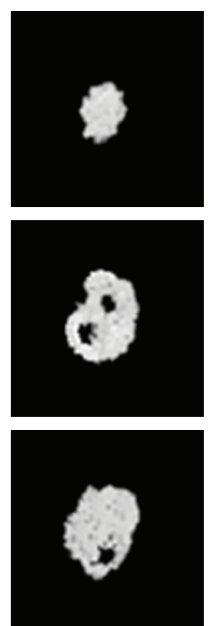
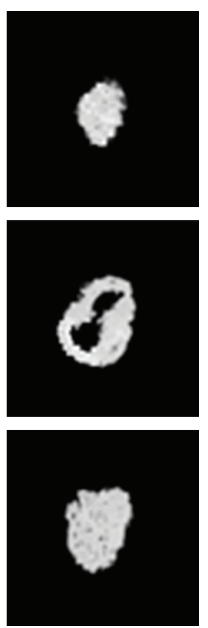

(b)

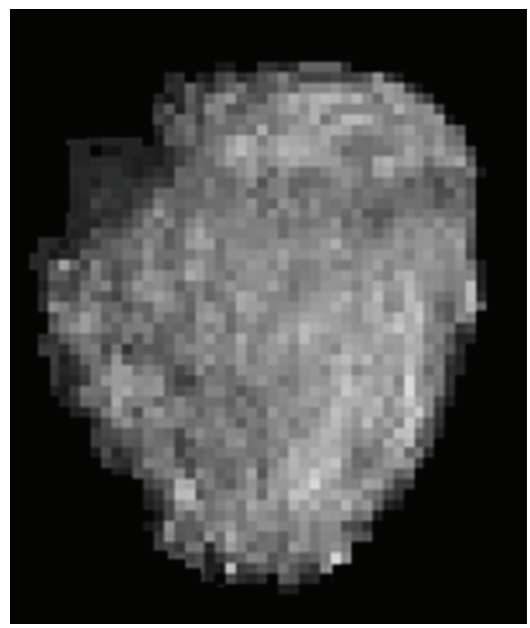

Figura 3. Exame PET real. (a) Fatias 2D das projeções das cavidades do miocárdio; (b) Volume gerado por meio de ferramentas de reconstrução do ImageJ.

Figure 3. Real PET test. (a) 2D slices of the projections of the myocardium cavities; (b) Volume generated by means of ImageJ reconstruction tools. 
Com o objetivo de avaliar imagens próximas às imagens reais, é vital que as imagens inicialmente adquiridas adotem as principais características de uma imagem PET real tal como o ruído Poisson próprio das imagens de Medicina Nuclear na modalidade PET. Para esse fim, foi incorporado computacionalmente ruído Poisson sobre todos os volumes de entrada através de um algoritmo realizado em MatLab por meio da função imnoise[image, 'Poisson']. Para verificar e/ou avaliar estatisticamente o tipo de ruído incorporado, um método denominado 'Chi-Square Goodness-of-Fit Test' (Nist/Sematech, 2011) foi utilizado.

Detalhes da implementação que incorpora aleatóriamente o ruído Poisson nos volumes simulados, assim como os pormenores do teste 'Chi-Square Goodness-of-Fit Test', podem ser visualizado em Flórez Pacheco (2012).

\section{Pré-processamento: filtragem de imagens $3 D$}

Podemos definir o ruído numa imagem digitalizada, como toda flutuação indesejável das intensidades dos pixels que pode comprometer tanto a sensibilidade quanto à qualidade da imagem (Sarmento et al., 2004). Uma das técnicas de processamento comumente utilizadas para melhorar a qualidade de uma imagem é a aplicação de algoritmos que permitam eliminar ruídos indesejáveis na imagem, geralmente gerados no processo de aquisição (Gonzalez e Woods, 2008). O objetivo deste processo de filtragem é conseguir uma imagem resultante com características mais adequadas que a imagem original para uma aplicação específica (Marques Filho e Vieira, 1999). A restauração de uma imagem através da remoção do ruído contido nela é essencial em aplicações com imagens médicas, e consiste basicamente em utilizar informações conhecidas a priori sobre as degradações da imagem, de modo que seja possível aplicar um processo inverso para remover essas distorções a fim de melhorar e recuperar os detalhes anatômicos que podem estar escondidos nos dados (Rodrigues et al., 2008).

Num processo de filtragem, o valor de intensidade de uma imagem depende não só da intensidade original, mas dos valores de intensidade de sua vizinhança. O tamanho desta vizinhança não é limitado, mas normalmente os vizinhos mais próximos têm maior influência que aqueles que estão mais distantes. Por se tratar de um ruído aleatório, a modelagem do ruído quântico é baseada apenas em ferramentas estatísticas. Os métodos de filtragem abordados, analisados, implementados e discutidos neste projeto são: a) Filtro da Mediana 3D; b) Filtro da Gaussiana Ponderada 3D; e c) Filtro de Anscombe/Wiener 3D.

\section{a) Filtro da Mediana $3 D$}

O filtro da Mediana é um filtro não linear, bastante comum, que realiza uma transformação baseado nas estatísticas locais com o propósito de suavizar ruídos do tipo impulsivo em imagens digitais. Este método de filtragem é bastante utilizado em aplicações onde é preciso filtrar o ruído e ao mesmo tempo preservar as bordas e os detalhes característicos da imagem (Romualdo, 2009). Basicamente o que esta técnica faz é substituir a intensidade do pixel central da janela pela mediana dos pixels situados em sua vizinhança, a qual vem definida pela dimensão da máscara escolhida (Marques Filho e Vieira, 1999). O filtro da Mediana 3D foi aplicado sob os volumes analisados partindo de três tamanhos de janela ou máscara diferentes: $3 \times 3 \times 3,5 \times 5 \times 5$ e $7 \times 7 \times 7$.

A Figura 4 mostra os resultados da filtragem aplicado sob o phantom do Ventrículo Esquerdo originado com o NCAT-4D. No entanto, em Flórez Pacheco (2012) são apresentados também resultados qualitativos aplicados sob a cavidade do Ventrículo Esquerdo simulado.

\section{b) Filtro da Gaussiana Ponderada 3D}

O filtro da Gaussiana Ponderada é um filtro linear, que usa máscaras de convolução para suavizar o ruído contido numa imagem digital. A filtragem de imagens usando o conceito da Gaussiana Ponderada se comporta como uma função matemática aplicada aos dados do perfil, expressada pela seguinte equação:

$$
f(x)=\left[\frac{1}{\sigma \sqrt{2 . \pi}}\right] \cdot e^{-\frac{1}{2}\left(\frac{x}{\sigma}\right)^{2}}
$$

onde $\sigma$ é o desvio padrão da distribuição com valor sigma considerado no processo da filtragem. $\mathrm{O}$ filtro gaussiano tem uma função interpoladora, que ameniza a amplitude do sinal dando mais suavidade aos contornos da superfície (Pertusa, 2003; Whitehouse, 1998). Esse filtro realiza uma média ponderada do perfil, onde os valores dos pesos se distribuem na forma gaussiana (distribuição normal). Foram testados seis diferentes valores de sigma: $\sigma=0,5, \sigma=1,0$, $\sigma=1,5, \sigma=2,0, \sigma=2,5$ e $\sigma=3,0$, que constituem os valores adequados dentro da curva de distribuição normal para a suavização. Na Figura 5 se mostram os resultados qualitativos da aplicação do filtro da Gaussiana Ponderada 3D sob o phantom do Ventrículo Esquerdo originado com o NCAT-4D.

\section{c) Filtro de Anscombe/Wiener $3 D$}

O principal método de filtragem aplicado neste trabalho efetua uma restauração nas imagens utilizando, numa primeira etapa, a Transformada de Anscombe (AT) e o filtro de Wiener pontual (Lee) para redução do ruído quântico. Posteriormente, é utilizada a Transformada Inversa de Anscombe (IAT) para o realce das estruturas de interesse da imagem filtrada. 


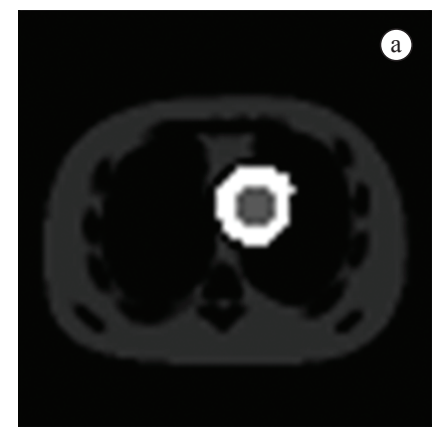

Original

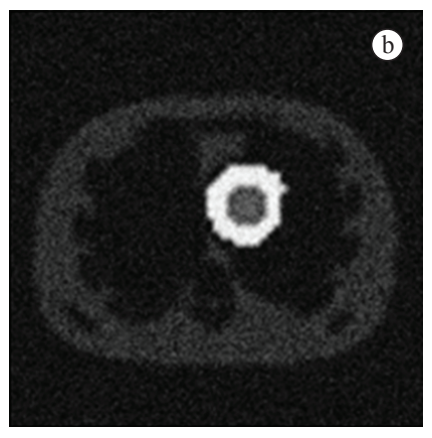

Com ruído

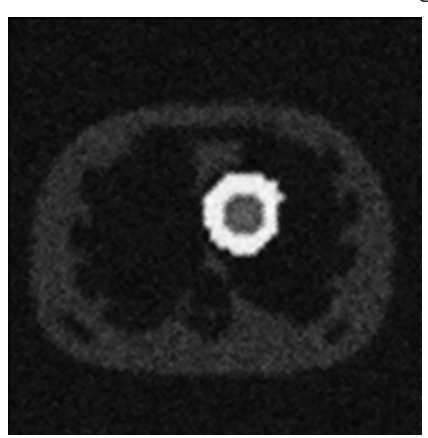

Janela $3 \times 3 \times 3$

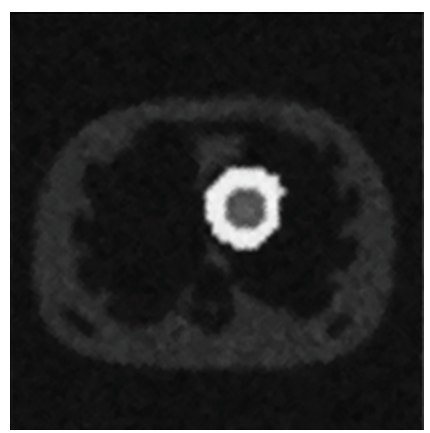

Janela $5 \times 5 \times 5$

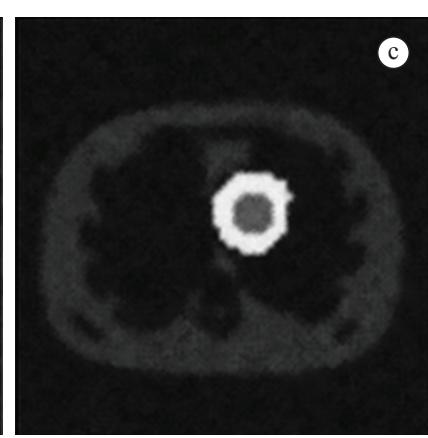

Janela $7 \times 7 \times 7$

Figura 4. (a) Imagem de uma fatia do phantom gerado através do NCAT-4D; (b) Fatia com ruído Poisson incorporado; e (c) Resultados qualitativos do filtro da mediana 3D sobre o ruído Poisson com três tamanhos de janelas diferente.

Figure 4. (a) Image of a slice of the phantom generated by NCAT-4D; (b) Slice with incorporated Poisson noise; and (c) Qualitative results of the $3 D$ median filter on the Poisson noise with three different window sizes.

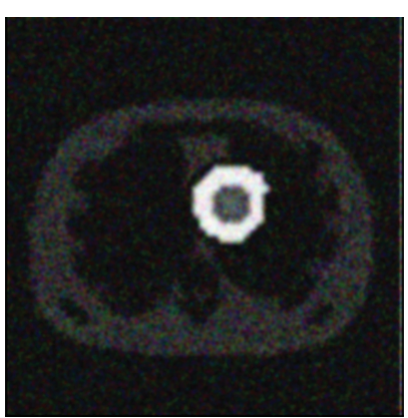

$\sigma=0,5$

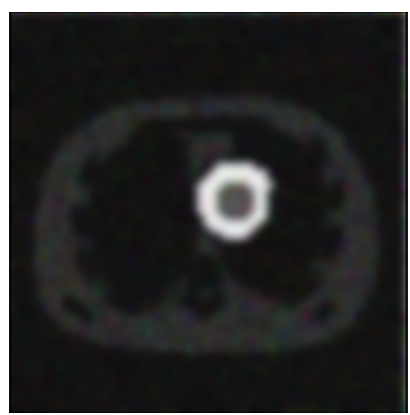

$\sigma=2,0$

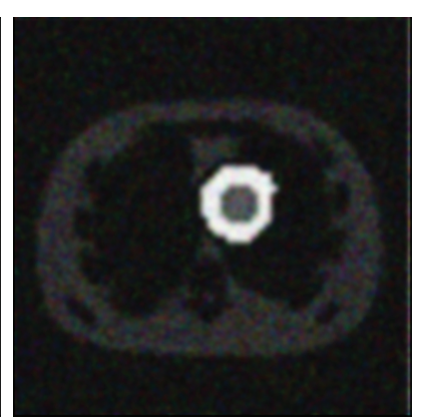

$\sigma=1,0$

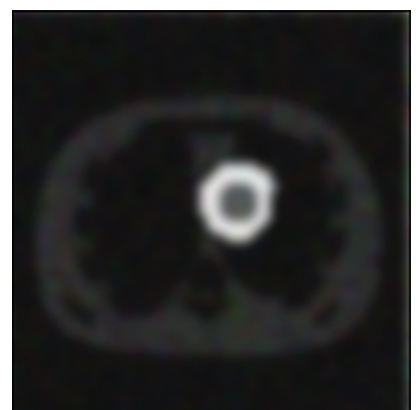

$\sigma=2,5$

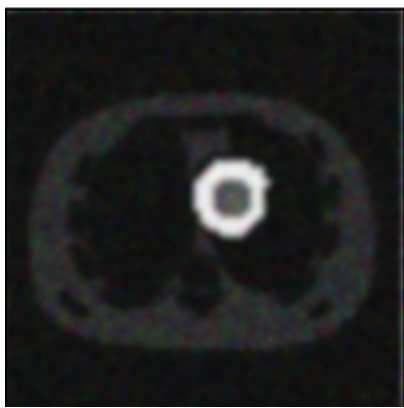

$\sigma=1,5$

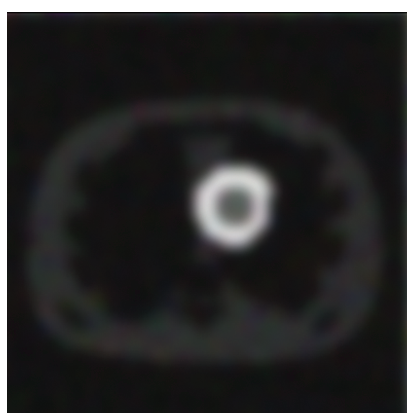

$\sigma=3,0$

Figura 5. Resultados qualitativos do filtro da Gaussiana ponderada 3D sobre o ruído Poisson do phantom gerado por meio do NCAT-4D, usando seis diferentes valores de sigma.

Figure 5. Qualitative results of the 3D weighted Gaussian filter on the Poisson noise of the phantom generated by means of NCAT-4D, using six different values of sigma. 
Transformada de Anscombe (AT) - É uma transformação não linear que permite transformar o ruído quântico de uma imagem digital, que é dependente do sinal, em um ruído aproximadamente independente do sinal, aditivo, Gaussiano, com média zero e variância unitária (Inouye, 1971; Romualdo, 2009).

De acordo com Anscombe (1948), dada a variável aleatória $\tilde{U}_{\mathrm{i}}$, com distribuição estatística de Poisson, podemos expressar a AT desta variável aleatória, utilizando a Equação 2:

$$
\tilde{Z}_{\mathrm{i}}=2 \cdot \sqrt{\tilde{U}_{\mathrm{i}}+\frac{3}{8}}
$$

Esta nova variável $\tilde{Z}_{i}$, poder ser representada por meio de um modelo aditivo (Inouye, 1971), conforme mostra a Equação 3:

$$
\tilde{Z}_{\mathrm{i}}=2 \cdot \sqrt{\tilde{U}_{\mathrm{i}}+\frac{1}{8}}+\tilde{N}_{\mathrm{i}}=\tilde{S}_{\mathrm{i}}+\tilde{N}_{\mathrm{i}}
$$

sendo $\tilde{N}_{i}$ um ruído aproximadamente independente do sinal $\tilde{S}_{i}$, descrito por uma distribuição Gaussiana com média zero e variância unitária. Após esta transformação, podem-se utilizar técnicas bem conhecidas aplicadas à nova variável, para a redução do ruído, que agora é aditivo e independente do sinal (Mascarenhas et al., 1999).

Filtro de Wiener pontual (Lee) - Sob o critério do mínimo erro quadrático médio, o Filtro de Wiener pontual (Lee, 1980) é um filtro linear ótimo e pode ser utilizado para a filtragem do ruído aditivo gaussiano. Assim, dado o vetor de observações $u$, para cada $u_{i}$, a Transformada de Anscombe é agora expressada pela Equação 4:

$\mathrm{z}_{\mathrm{i}}=2 \cdot \sqrt{\mathrm{u}_{\mathrm{i}}+\frac{3}{8}}$

Obtém-se uma estimativa sem ruído $\hat{\mathrm{s}}_{\mathrm{i}}$ para $\mathrm{z}_{\mathrm{i}}$ utilizando o Filtro de Wiener pontual dado pela Equação 5:

$\hat{S}_{\mathrm{i}}=\mathrm{E}\left[\tilde{S}_{\mathrm{i}}\right]+\frac{\sigma_{\hat{S}_{\mathrm{i}}}^{2}}{\sigma_{\hat{S}_{\mathrm{i}}}^{2}+1}\left(\mathrm{z}_{\mathrm{i}}-\mathrm{E}\left[\tilde{S}_{\mathrm{i}}\right]\right)$

sendo que a média e a variância de $\hat{\mathrm{s}}_{\mathrm{i}}$, expressas respectivamente por $E[\tilde{S i}]$ e $\sigma_{\hat{s}_{i}}^{2}$, são medidas locais e podem ser estimadas na prática a partir da imagem a ser filtrada.

Transformada Inversa de Anscombe (IAT) - Após a filtragem do ruído, aplicamos a transformação inversa de Anscombe para termos uma estimativa da imagem degradada sem o ruído quântico (Romualdo, 2009). Podemos expressar a IAT sobre $\hat{s}_{\mathrm{i}}$ pela Equação 6: $\hat{b}_{\mathrm{i}}=\frac{1}{4} \cdot \hat{s}^{2}-\frac{1}{8}$

$\mathrm{Na}$ Figura 6, mostram-se duas fatias bidimensionais similares de uma porção da cavidade do Ventrículo Esquerdo simulado. A primeira fatia contem ruído quântico tipo Poisson, enquanto a outra é a fatia resultante depois da execução do filtro Anscombe/Wiener. Analisando o perfil de cada uma delas, através da função $<$ Profile $>$ do ImageJ, pode-se distinguir a considerável eliminação do ruído Poisson. Este processo foi aplicado sobre o conjunto de fatias que conformam o volume examinado.

\section{Segmentação de imagens digitais}

A segmentação é um processo que permite selecionar regiões específicas dentro de um grupo de objetos que compõem uma imagem (Gonzales e Woods, 2008). Na literatura encontramos uma variedade de métodos para este fim, entre os mais conhecidos pode-se mencionar os seguintes: Snakes (Kass et al., 1988; Xu, 1998), Level Set (Osher e Fedkiw, 2002), Fuzzy Connectedness (Nyúl et al., 2002; Pednekar; Kakadiaris, 2006; Udupa e Saha, 2003), IFT (Falcão et al., 2004), Region Growing (Gonzalez e Woods, 2008), Thresholding (Gonzalez e Woods, 2008; Otsu, 1979), entre outras. As investigações neste trabalho têm como objetivo analisar e aplicar algoritmos de segmentação baseados no conceito de Fuzzy Connectedness. O principal conceito da Teoria Fuzzy é o fato de dar flexibilidade na modelagem, permitindo desenvolver algoritmos semelhantes ao pensamento humano (Souza e De Oliveira, 1992).

O método Fuzzy Connectedness tem se mostrado eficiente na segmentação de imagens médicas, mesmo comparado com outros métodos (Kass et al., 1988; Xu, 1998), e dependendo da aplicação, ele é mais interessante, pois é um método robusto, principalmente quando se trata de imagens com intensidades não-uniformes (Pednekar e Kakadiaris, 2006).

As principais definições que englobam a Teoria Fuzzy, entre os quais se destacam o Espaço Digital Fuzzy, Cena de Pertinência, Adjacência, Afinidade, Conectividade e Objeto Fuzzy, são descritos em Flórez Pacheco (2012). Baseados nestes conceitos, pode-se estabelecer uma classificação de relação entre os elementos que pertencem, ou não, a um objeto específico (Nyúl et al., 2002; Udupa e Samarasekera, 1996; Udupa e Saha, 2003).

Partindo da adjacência e afinidade fuzzy, principais conceitos da segmentação usando Fuzzy Connectedness, é possível encontrar uma conectividade entre quaisquer dois spels, c e d, no domínio da cena $\mathrm{C}$, a partir de uma série de afinidades locais 

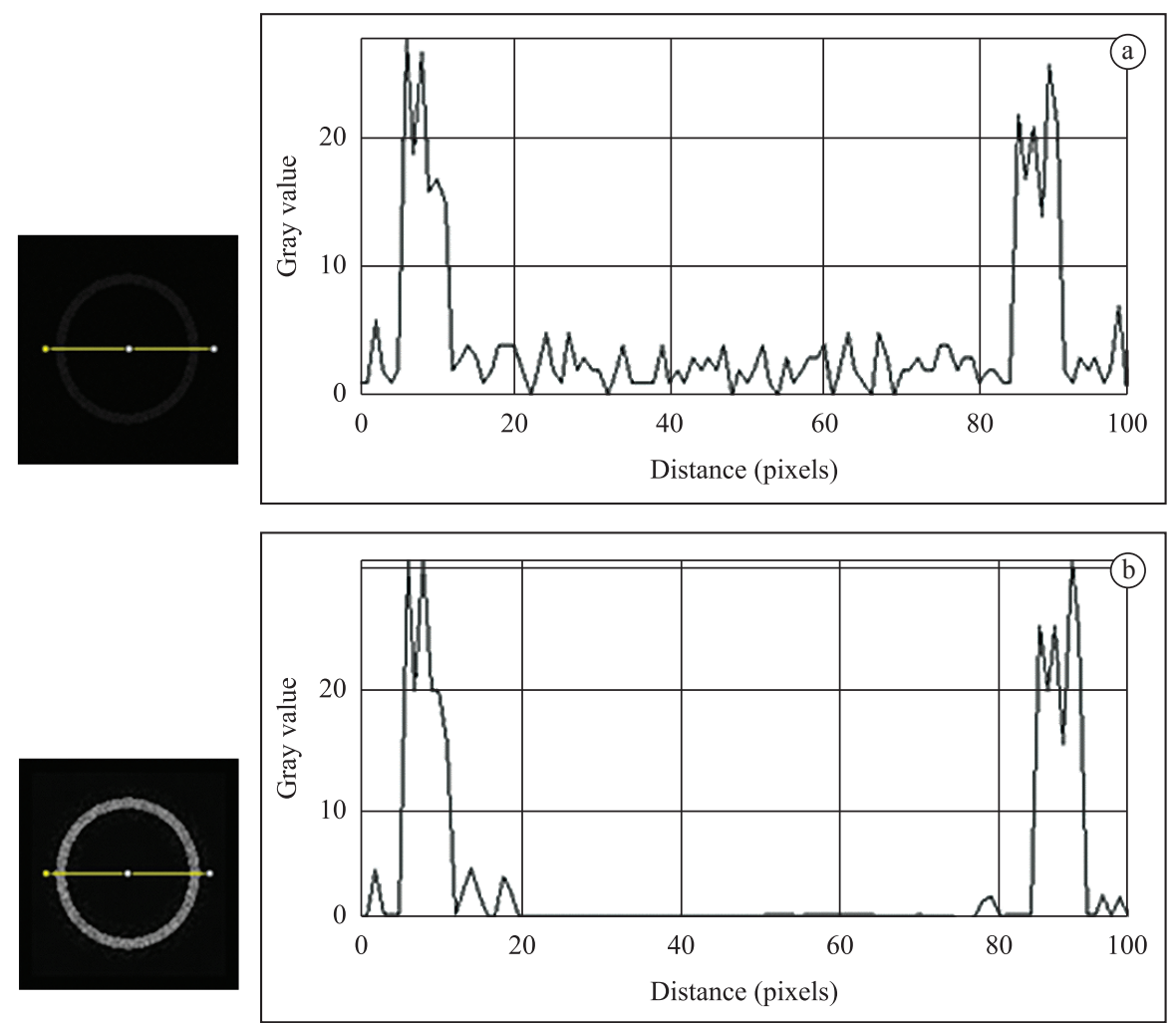

Figura 6. Perfil (linha central horizontal) de (a) Imagem com ruído Poisson e (b) Imagem filtrada

Figure 6. Profile (horizontal central line) of (a) Image with Poisson noise and (b) Image filtered.

entre eles (Nyúl et al., 2002). Neste ponto, fica claro que existem vários caminhos passando somente por adjacências unitárias que consigam unir esses dois spels. Assumindo que $\mathrm{P}_{\mathrm{c}, \mathrm{d}}=\left(\mathrm{s}_{1}, \mathrm{~s}_{2}, \ldots, \mathrm{s}_{\mathrm{n}}\right)$ seja um caminho entre $s_{1}=c$ e $s_{\mathrm{N}}=d$, onde $s_{i}$ são $\mathrm{N}$ spels adjacentes em pares entre si, a afinidade (mais fraca) que melhor representa este caminho está dada por:

$\mu_{\mathrm{K}}\left(\mathrm{p}_{\mathrm{c}, \mathrm{d}}\right)=\min _{1<i<N}\left(\mu_{\mathrm{k}}\left(\mathrm{s}_{\mathrm{i}-1}, \mathrm{~s}_{\mathrm{i}}\right)\right)$

Como foi dito, são inúmeros os caminhos entre dois spels e como é evidente num caso ótimo, a conectividade global deverá considerá-los todos. Com tudo isso, a seleção da maior afinidade de caminho será a melhor representação da conectividade global. Portanto, assumindo que $\mathrm{P}_{\mathrm{c}, \mathrm{d}}$ seja um conjunto de todos os caminhos $\mathrm{p}$, a conectividade global entre $\mathrm{c}$ e d é definida como:

$\mu_{\mathrm{K}}(\mathrm{c}, \mathrm{d})=\max _{\mathrm{p} \in \mathrm{P}_{\mathrm{c}, \mathrm{d}}}\left(\min _{1<i<N}\left(\mu_{\mathrm{k}}\left(\mathrm{s}_{\mathrm{i}-1}, \mathrm{~s}_{\mathrm{i}}\right)\right)\right)$

Faz-se necessário agora adentrarmos na estrutura para o cálculo da Conectividade. Em Gonzalez e Woods (2008), a Afinidade está conformada por três propriedades fundamentais: adjacência $\left(\mu_{\alpha}\right)$, homogeneidade $\left(\mu_{\psi}\right)$, e intensidade $\left(\mu_{\phi}\right)$. Tendo em consideração que a relação de adjacência $\mu_{\alpha}(\mathrm{c}, \mathrm{d})$ indica a proximidade espacial entre os elementos avaliados, sendo que se c e d são vizinhos a adjacência Fuzzy é unitária. A afinidade fica expressada como mostra a Equação 9:

$\mu_{\mathrm{k}}=\mathrm{g}\left(\mu_{\psi}(\mathrm{c}, \mathrm{d}), \mu_{\phi}(\mathrm{c}, \mathrm{d})\right)$

Finalmente, o cálculo de afinidade para cada par de spels c e d de um caminho $\mathrm{P}_{\mathrm{c}, \mathrm{d}}$ pode se definido pela seguinte expressão:

$\mu_{\mathrm{k}}=\mathrm{w}_{1} \cdot \mu_{\psi}(\mathrm{c}, \mathrm{d})+\mathrm{w}_{2} \cdot \mu_{\phi}(\mathrm{c}, \mathrm{d})$

onde $\mathrm{w}_{1}$ e $\mathrm{w}_{2}$ são valores numéricos não negativos que representam os pesos das componentes de homogeneidade e intensidade, respectivamente. Expressões numéricas que permitem o cálculo dos valores que compõem o termo de homogeneidade $\left(\mu_{\psi}\right)$, e intensidade $\left(\mu_{\phi}\right)$ são indicados e detalhados em Flórez Pacheco (2012). Em Nyúl et al. (2002) os pesos $\mathrm{w}_{1}$ e $\mathrm{w}_{2}$ são expressos da seguinte maneira: 
$\mathrm{w}_{2}=\frac{\mu_{\phi}(\mathrm{c}, \mathrm{d})}{\mu_{\psi}(\mathrm{c}, \mathrm{d})+\mu_{\phi}(\mathrm{c}, \mathrm{d})}, \mathrm{w}_{1}=1-\mathrm{w}_{2}$

Neste projeto quatro diferentes abordagens de segmentação foram implementadas, os quais correspondem aos principais métodos abordados em Nyúl et al. (2002) e Udupa e Saha (2003), sendo elas as seguintes:

Algoritmo Genérico - É o método de segmentação mais básico proposto em Nyúl et al. (2002) e Udupa e Saha (2003). Precisa-se definir uma semente (seed) que pertence ao objeto de interesse que vai se segmentar. A partir desse instante, são calculados os valores de adjacência, afinidade e conectividade de cada um dos spels da imagem com respeito à semente definida. Estes valores são armazenados numa tabela e baseado numa relação ou grau de pertinência estabelecido, é definido quais dos elementos analisados pertencem e quais não ao objeto de interesse requerido. Esta abordagem utiliza uma manipulação dos dados proposta por Dijkstra (Dijkstra, 1959; Nyúl et al., 2002).

Algoritmo LIFO - Esta abordagem mantém o enfoque do algoritmo Genérico, no entanto, introduz uma importante variante baseada em adotar um método mais eficiente de administrar as estruturas de dados com os valores de cada um dos parâmetros envolvidos no processo de segmentação (Nyúl et al., 2002). Especificamente neste caso, a manipulação dos valores armazenados na tabela, está regida pelo conceito de Dial (Dial, 1969) e utiliza a estrutura de dados LIFO (do inglês: last input-first output).

Algoritmo kTetaFOEMS - Este algoritmo, consiste basicamente em identificar um objeto fuzzy $0_{\mathrm{k} \theta}(\mathrm{s})$ definido em $\mathrm{C}$, definindo-se a priori um valor limiar $\theta$ através do qual se estabeleça uma pertinência mínima de tal forma que para uma semente qualquer $\mathrm{s} \in \mathrm{S}$, se cumpram as condições seguintes (Lage, 2010; Udupa e Saha, 2003): $\mathrm{s} \in 0_{\mathrm{k} \theta}(\mathrm{s}) ; \forall \mathrm{c}, \mathrm{d} \in \mathrm{O}_{\mathrm{K} \theta}(\mathrm{s}), \mu_{\mathrm{K}}(\mathrm{c}, \mathrm{d}) \geq \theta$; $\forall \mathrm{c} \in \mathrm{O}_{\mathrm{K} \theta}(\mathrm{s})$ e $\mathrm{d} \notin \mathrm{O}_{\mathrm{K} \theta}(\mathrm{s}), \mu_{\mathrm{K}}(\mathrm{c}, \mathrm{d})<\theta$. Finalmente como resultado da segmentação, é obtido um objeto Fuzzy $0_{\mathrm{k} \theta}(\mathrm{s})$ em C.

É importante especificar que os pesos usados nestas três primeiras abordagens de segmentação, foram definidos para valores de $\mathrm{w}_{1} \mathrm{e}_{2}$ iguais a 0,5 . No entanto, em Flórez Pacheco (2012) foi utilizado um critério denominado analise das Curvas Características de Operação do Receptor (ROC) que permitiu obter os pesos adequados $\left(\mathrm{w}_{1}=0,3 \mathrm{e}_{2}=7\right)$ que ofereceram os melhores resultados de segmentação.

Algoritmo Pesos Dinâmicos - A particularidade desta abordagem, em relação aos três algoritmos de segmentação anteriores, consiste em realizar ajustes dinâmicos dos pesos $\mathrm{w}_{1} \mathrm{e}_{2}$ através das igualdades da Equação 11, que relacionam os componentes de homogeneidade e intensidade, para cada par de spels (c, d) de um caminho $\mathrm{P}_{\mathrm{c}, \mathrm{d}}$ (Nyúl et al., 2002; Udupa e Saha, 2003).

Na Figura 7 é representado o processo de segmentação de uma fatia 2D do Ventrículo Esquerdo dentro da caixa torácica através do algoritmo 3D que utiliza Pesos Dinâmicos, tendo como objeto de interesse o Ventrículo Esquerdo do Coração. O processo de segmentação foi realizado sob os volumes que foram previamente filtrados usando o Filtro da Mediana $3 \mathrm{~d}$ com janela $5 \times 5 \times 5$, o Filtro da Gaussiana Ponderada 3D usando $\sigma=1,5$ e o Filtro Anscombe/Wiener, respectivamente.

\section{Avaliação dos resultados}

Constitui uma etapa de muita importância dentro da pesquisa, já que representa um processo que vai nos permitir conhecer o nível de eficiência, acurácia, e precisão dos algoritmos, tanto de filtragem quanto de segmentação, utilizados ao longo deste trabalho. Seleccionou-se uma abordagem, descrita em Udupa et al. (2002), cuja solução se apresenta mais completa em relação ao presente projeto. Esta análise consiste basicamente em usar os parâmetros comparativos Verdadeiro Positivo (TP) e Falso Positivo (FP), de acordo às seguintes relações:

$\mathrm{TP}=\frac{\mathrm{A}}{\mathrm{C}}$

$\mathrm{FP}=\frac{\mathrm{B}}{\mathrm{C}}$

sendo C a segmentação padrão (gold standard), A representa a intersecção da segmentação padrão com a segmentação obtida, B representam a diferença da segmentação padrão com a segmentação processada.

Além destes dois parâmetros, incorporou-se um terceiro parâmetro para dar maior consistência a esta etapa de avaliação. Trata-se da Máxima Distância $\left(\operatorname{Max}_{\text {dist }}\right)$ calculado entre o contorno da imagem padrão (Cont $)$ e o contorno da imagem segmentada (Cont s (Moraes e Furuie, 2011). Este parâmetro nos permite ter uma estimação do máximo desvio existente entre as duas imagens comparadas. Este parâmetro está representado pela seguinte equação:

$\operatorname{Max}_{\text {dist }}=\max \left|\operatorname{Cont}_{\mathrm{P}}-\operatorname{Cont}_{\mathrm{S}}\right|$

No caso das imagens simuladas do Ventrículo Esquerdo, as imagens padrões serão as imagens geradas no processo inicial (antes da incorporação do ruído Poisson). Já para os phantoms gerados pelo NCAT-4D, uma vez que a imagem original representava o Ventrículo Esquerdo junto com a caixa 

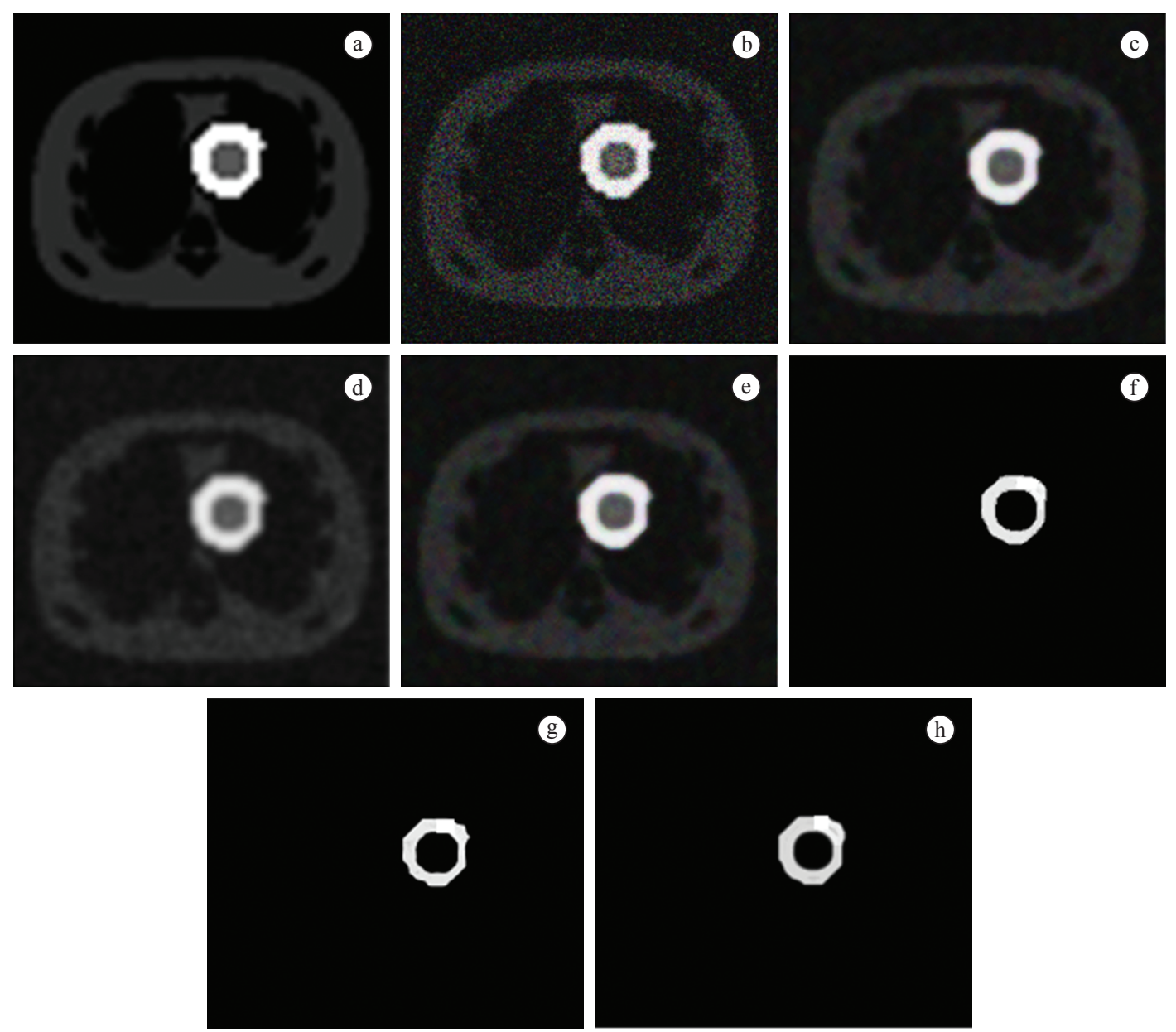

Figura 7. a) Fatia 2D de uma secção do Ventrículo Esquerdo dentro da estrutura da caixa torácica simulada usando o NCAT-4D; b) Fatia 2D com ruído Poisson incorporado computacionalmente; Fatias 2D após a etapa de filtragem através do c) Filtro da Mediana 3D com janela $5 \times 5 \times 5$; d) Filtro da Gaussiana Ponderada 3D com $\sigma=1,5$; e) Usando a Transformada de Anscombe e o Filtro de Wiener; (f), (g) e (h) Fatias 2D obtidas depois do processo de Segmentação utilizando o algoritmo com Pesos Dinâmicos, tendo como entrada as imagens filtradas com o Filtro da Mediana 3D, Filtro da Gaussiana Ponderada 3D e o Filtro Anscombe/Wiener, respectivamente.

Figure 7. a) $2 D$ slice of the left ventricle within the rib cage simulated using the NCAT-4D; (b) $2 D$ slice with Poisson noise computationally incorporated; $2 D$ slices after the filtering process by (c) $3 D$ Median filter with $5 \times 5 \times 5$ window; (d) $3 D$ weighted Gaussian filter with $\sigma=1.5 ; e)$ With the Anscombe/Wiener filter; $f$ ), g) and h) $2 D$ slices obtained after the segmentation process using the Dynamic Weights algorithm, for the filtered images with the 3D Median filter, the $3 D$ Weighted Gaussian filter and the Anscombe/Wiener filter, respectively.

torácica, uma etapa intermediária de segmentação (por meio de limiarização) foi realizada para capturar unicamente o objeto de estudo, que é o Ventrículo Esquerdo. O produto desta operação será nossa imagem gold standard que será comparada com as imagens processadas por meio do procedimento de avaliação definido.

Já para o caso das imagens reais, não foi possível submeter estes volumes na estrutura de processamento implementada no projeto nem aplicar o processo de avaliação ao não contar com as imagens gold standard. Neste caso em particular, foi realizada a etapa de quantificação dos voxels de cada volume analisado, conforme explicado a seguir.

\section{Quantificação dos volumes}

O processo de quantificação consiste em contabilizar cada um dos voxels que pertencem ao objeto de interesse em cada um das fatias que conformam o volume analisado. A quantificação dos volumes é uma tarefa rápida, simples e bastante direta, já que os objetos de interesse ficaram bem definidos após a etapa de segmentação. Este procedimento foi aplicado sobre os volumes PET reais, cujas aquisições foram geradas em frames num período de tempo determinado. No entanto, é importante indicar que não foi possível efetivar uma avaliação mais objetiva devido à falta de uma referência padrão (gold standard) dos volumes PET reais analisados. 
Os volumes pertencentes ao exame PET real estão conformados por um conjunto de 36 volumes com 35 cortes ou fatias cada. Para propósitos do projeto foram analisados unicamente os quatro últimos volumes.

Todas as imagens analisadas tem extensão DICOM, entendendo por imagem DICOM aquela imagem radiológica que possui uma formatação padrão global que permite uma transmissão de informação médica num formato eletrônico estruturado e dentro de um protocolo definido, com o qual foi possível conhecer a dimensão exata de cada elemento da imagem e, portanto, obter a quantificação do volume do objeto de interesse tanto em unidades de voxels quanto $\mathrm{em}^{\mathrm{mm}^{3}}$.

Conforme indicado, cada um dos volumes reais analisados está constituído por 35 fatias de $128 \times 128$ pixels que equivalem a $220 \times 220 \mathrm{~mm}$. Fazendo os cálculos, obtém-se que cada pixel é igual a $2.95 \mathrm{~mm}^{2}$. Sendo conhecido o espaçamento entre cada uma das fatias que é igual a $4.25 \mathrm{~mm}$, fica determinado que cada voxel é equivalente a $12.55 \mathrm{~mm}^{3}$. Com esta equivalência foi possível obter os valores de quantificação requeridos. Mais detalhes deste procedimento podem ser consultados em Flórez Pacheco (2012).

\section{Resultados}

Para cada um dos 30 volumes do Ventrículo Esquerdo simulado e para o volume representativo do phantom gerado através do NCAT-4D, foram testados os três algoritmos de filtragem com cada um dos quatro algoritmos de segmentação implementados. Vale a pena relembrar que o filtro da Mediana 3D foi testado através de três diferentes tamanhos de janelas
$(3 \times 3 \times 3,5 \times 5 \times 5$ e $7 \times 7 \times 7)$, e o filtro da Gaussiana ponderada $3 \mathrm{D}$ através de seis diferentes valores de sigma $(\sigma=0,5, \sigma=1,0, \sigma=1,5, \sigma=2,0, \sigma=2,5 \mathrm{e}$ $\sigma=3,0)$. Portanto, foram testadas e avaliadas quarenta configurações distintas de filtragem e segmentação. Este procedimento permitiu conhecer, por um lado os parâmetros específicos que promoviam obter um filtro com maior eficiência e, por outro lado, determinar qual par (filtro mais segmentador) representava a via mais adequada para o processamento das imagens analisadas.

A Tabela 1 apresenta os resultados de todas as combinações de filtragem seguidas da etapa de segmentação, aplicado no phantom NCAT-4D. Cabe ressaltar que, no caso do Filtro da Mediana 3D, a filtragem usando janela $5 \times 5 \times 5$ apresentou melhores resultados frente aos outros dois tamanhos de janela. No caso do Filtro da Gaussiana Ponderada $3 \mathrm{D}$, usando o valor de sigma igual a 1,5 foi possível obter melhores resultados que com os outros cinco valores de sigma testados.

Partindo dos resultados dos testes realizados na Tabela 1, foi elaborado um teste adicional considerando a etapa de filtragem usando o Filtro Anscombe/Wiener 3D com cada uma das quatro abordagens diferentes de segmentação 3D. Note-se que os testes foram realizados em três estágios diferentes: imagens padrão (tal como foram simuladas), imagens com ruído Poisson (sem ter aplicado nenhum filtro) e imagens após a etapa de filtragem. Na Tabela 2 são mostrados os valores dos três parâmetros de avaliação utilizados neste trabalho, para cada uma das doze combinações testadas.

Resultados de todos estes processos, realizados sob os 30 volumes do Ventrículo Esquerdo simulados, são mostrados em Flórez Pacheco (2012).

Tabela 1. Quadro comparativo dos resultados dos algoritmos de filtragem versus segmentação para o phantom representativo gerado através do NCAT-4D.

Table 1. Comparative table of the results of the filtering versus segmentation algorithms that correspond to the representative NCAT-4D phantom generated by NCAT-4D.

\begin{tabular}{|c|c|c|c|c|c|c|c|c|c|}
\hline \multirow{3}{*}{\multicolumn{2}{|c|}{ Filtros 3D }} & \multicolumn{8}{|c|}{ Segmentadores } \\
\hline & & \multicolumn{2}{|c|}{ Genérico } & \multicolumn{2}{|c|}{ Lifo } & \multicolumn{2}{|c|}{ Kteta $(0,10)$} & \multicolumn{2}{|c|}{ Pesos dinâmicos } \\
\hline & & TP\% & FP\% & TP\% & FP\% & ТP\% & FP\% & ТP\% & FP\% \\
\hline \multirow{3}{*}{ Mediana } & $3 \times 3 \times 3$ & 90,39 & 4,81 & 90,53 & 4,63 & 90,87 & 4,19 & 91,24 & 4,02 \\
\hline & $5 \times 5 \times 5$ & 91,11 & 3,63 & 91,43 & 3,58 & 91,58 & 3,81 & 91,92 & 3,45 \\
\hline & $7 \times 7 \times 7$ & 88,48 & 5,92 & 88,52 & 5,70 & 88,92 & 5,35 & 89,33 & 5,11 \\
\hline \multirow{6}{*}{$\begin{array}{l}\text { Gaussiana } \\
\text { ponderada }\end{array}$} & Sigma 0,5 & 88,56 & 5,85 & 88,75 & 5,77 & 89,22 & 5,44 & 89,37 & 5,30 \\
\hline & Sigma 0,1 & 89,88 & 4,30 & 90,04 & 4,24 & 90,34 & 4,28 & 90,77 & 4,12 \\
\hline & Sigma 1,5 & 91,45 & 3,32 & 91,71 & 3,02 & 91,80 & 3,00 & 92,23 & 2,91 \\
\hline & Sigma 2,0 & 89,49 & 4,74 & 89,62 & 4,24 & 89,87 & 4,17 & 90,22 & 4,12 \\
\hline & Sigma 2,5 & 88,74 & 5,79 & 88,89 & 5,72 & 89,35 & 5,48 & 89,59 & 5,21 \\
\hline & Sigma 3,0 & 88,08 & 6,22 & 88,46 & 5,96 & 88,86 & 5,43 & 89,26 & 5,37 \\
\hline \multicolumn{2}{|c|}{ Anscombe/Lee } & 92,12 & 2,51 & 92,58 & 2,11 & 93,09 & 2,23 & 94,05 & 2,31 \\
\hline
\end{tabular}


Com o conjunto de resultados obtidos nos testes feitos sobre todos os volumes simulados, foi possível realizar certas escolhas que delimitaram alguns parâmetros, através dos quais foram obtidas as quantificações dos volumes PET reais analisados.

$\mathrm{Na}$ Tabela 3 são apresentados os resultados de quantificação de um dos volumes PET reais (volume PET $\mathrm{N}^{\circ} 36$ ), os resultados de quantificação dos outros volumes de PET reais analisados, podem ser observados em Flórez Pacheco (2012). Note-se que temos considerado unicamente os testes de filtragem que representaram os melhores resultados, junto com cada um dos algoritmos de segmentação implementados no projeto.

Os valores de quantificação são representados em duas unidades de volume: voxels e $\mathrm{mm}^{3}$.

Uma das particularidades do processo de quantificação, foi a utilização dos valores otimizados dos pesos $\mathrm{w}_{1}=0,3$ e $\mathrm{w}_{2}=0,7$, nos algoritmos de segmentação 3D: Genérico, LIFO e KTetaFOEMS.

\section{Discussão}

Vários trabalhos já mostraram a efetividade do filtro de Anscombe/Wiener para imagens de Medicina Nuclear. O presente trabalho permitiu demonstrar e corroborar a supremacia do Filtro de Anscombe/Wiener em comparação aos outros filtros testados, tendo em consideração as características dos volumes PET analisados e os objetivos e/ou propósitos específicos deste projeto.

Comprovou-se que os algoritmos baseados na teoria Fuzzy Connectedness são bastante adequados para a Segmentação de imagens 3D, e facilmente escaláveis para n-dimensões. Apresentaram robustez e bons resultados quando testados nos diferentes volumes simulados, phantoms e volumes reais.

Dentro dos quatro algoritmos de segmentação utilizados, o algoritmo Fuzzy Connectedness que utiliza Pesos Dinâmicos apresentou melhores resultados. É importante indicar que esta abordagem mesmo que tenha apresentado um ótimo desempenho como segmentador

Tabela 2. Parâmetros de avaliação para diversas implementações do fuzzy connectedness no processo de segmentação das imagens padrão, com ruído e filtradas (usando o Filtro de Anscombe/Wiener) para o phantom NCAT-4D.

Table 2. Assessment parameters for different implementations of fuzzy connectedness in the segmentation process of standard, noisy and filtered images (using the Anscombe/Wiener filter) that correspond to the NCAT-4D phantom.

\begin{tabular}{ccccc}
\hline \multirow{2}{*}{ Algoritimos 3D } & $\begin{array}{c}\text { Parâmetros de } \\
\text { avaliação }\end{array}$ & Phantom NCAT-4D \\
\cline { 2 - 5 } & \%VP & Imagens padrão & Imagens com ruído & Imagens filtradas \\
\hline \multirow{2}{*}{ Genérico } & \%FP & $0,24 \pm 0,05$ & $89,55 \pm 6,75$ & $92,12 \pm 1,48$ \\
& MaxDist & 1 pixel & $6,75 \pm 1,84$ & $2,51 \pm 0,98$ \\
& \%VP & $99,38 \pm 0,04$ & $89,95 \pm 6,17$ & 2 pixel \\
\hline \multirow{2}{*}{ Lifo } & $\% \mathrm{FP}$ & $0,45 \pm 0,04$ & $6,45 \pm 1,12$ & $2,11 \pm 0,88$ \\
& MaxDist & 1 pixel & 5 pixel & 2 pixel \\
\hline \multirow{2}{*}{ KFOEMS- $\theta(\theta=0.10)$} & $99,40 \pm 0,05$ & $90,07 \pm 6,27$ & $93,09 \pm 1,81$ \\
& \%FP & $0,49 \pm 0,05$ & $6,05 \pm 1,48$ & $2,23 \pm 0,84$ \\
& MaxDist & 1 pixel & 5 pixel & 2 pixel \\
\hline \multirow{2}{*}{ Pesos dinâmicos } & \%VP & $99,64 \pm 0,03$ & $91,51 \pm 5,57$ & $94,05 \pm 1,32$ \\
& \%FP & $0,27 \pm 0,03$ & $5,34 \pm 1,23$ & $2,31 \pm 0,93$ \\
& MaxDist & 1 pixel & 5 pixel & 2 pixel \\
\hline
\end{tabular}

Tabela 3. Resultados da quantificação do volume PET real $\mathrm{N}^{\circ} 36$.

Table 3. Quantifying results of the real PET volume No. 36.

\begin{tabular}{|c|c|c|c|c|}
\hline Volume & Segmentador 3D & $\begin{array}{l}\text { Filtro de mediana 3D } \\
\quad(\text { janela } 5 \times 5 \times 5)\end{array}$ & $\begin{array}{l}\text { Filtro da Gauss } \\
\text { ponderada } 3 \mathrm{D} \text { (sigma } 1,5)\end{array}$ & $\begin{array}{c}\text { Filtro de Anscombe/ } \\
\text { Lee 3D }\end{array}$ \\
\hline \multirow{4}{*}{$\begin{array}{c}\text { Volume nro. } 36 \\
\text { Código - } 3030000\end{array}$} & Genérico & $\begin{array}{l}16550 \text { voxels } \\
\left(83081 \mathrm{~mm}^{-3}\right)\end{array}$ & $\begin{array}{l}17347 \text { voxels } \\
\left(80046 \mathrm{~mm}^{-3}\right) \\
\end{array}$ & $\begin{array}{l}92305 \text { voxels } \\
\left(92305 \mathrm{~mm}^{-3}\right)\end{array}$ \\
\hline & Lifo & $\begin{array}{l}18817 \text { voxels } \\
\left(94463 \mathrm{~mm}^{-3}\right)\end{array}$ & $\begin{array}{l}17597 \text { voxels } \\
\left(88339 \mathrm{~mm}^{-3}\right)\end{array}$ & $\begin{array}{l}19172 \text { voxels } \\
\left(96245 \mathrm{~mm}^{-3}\right)\end{array}$ \\
\hline & KTetaFOEMS & $\begin{array}{c}20070 \text { voxels } \\
\left(100751 \mathrm{~mm}^{-3}\right)\end{array}$ & $\begin{array}{c}21435 \text { voxels } \\
\left(107603 \mathrm{~mm}^{-3}\right)\end{array}$ & $\begin{array}{c}23805 \text { voxels } \\
\left(119501 \mathrm{~mm}^{-3}\right)\end{array}$ \\
\hline & Pesos dinâmicos & $\begin{array}{c}24787 \text { voxels } \\
\left(124433 \mathrm{~mm}^{-3}\right)\end{array}$ & $\begin{array}{c}24622 \text { voxels } \\
\left(123604 \mathrm{~mm}^{-3}\right)\end{array}$ & $\begin{array}{c}25642 \text { voxels } \\
\left(128597 \mathrm{~mm}^{-3}\right)\end{array}$ \\
\hline
\end{tabular}


tem associado um tempo computacional maior, com respeito com os tempos dos outros algoritmos. Esta diferença do tempo de processamento ocorre, pois o Algoritmo baseado em Pesos Dinâmicos faz um processo interno adicional com o propósito de obter os pesos $\mathrm{W}_{1}$ e $\mathrm{W}_{2}$ mais adequados para a etapa de segmentação.

Os resultados encontrados, com a utilização dessa metodologia, mostraram-se satisfatórios. Foi obtido, para o caso do Ventrículo Esquerdo simulado, 2,19\% de Falsos Positivos e 98,49\% de Verdadeiros Positivos no final da sequência de processamento instaurada, assim como 2,31\% de Falsos Positivos e 94,05\% de Verdadeiros Positivos para o phantom gerado por meio do NCAT-4D, utilizando para ambos casos na etapa de filtragem o Filtro de Anscombe/Wiener e na etapa de segmentação o algoritmo baseado em Pesos Dinâmicos. Nesse sentido, verificou-se a melhora do processo de segmentação através da etapa de filtragem com o uso do Filtro de Anscombe/Wiener.

Pelo fato de não dispor das imagens de referência padrão (gold standard) dos volumes PET reais, não foi possível submeter estes volumes na estrutura de processamento implementada no projeto. No entanto, usando as escolhas feitas ao longo do projeto, foi possível quantificar um conjunto de volumes PET reais.

\section{Conclusão}

Tendo em consideração as características dos volumes PET analisados e os objetivos e/ou propósitos específicos deste projeto, o presente trabalho mostrou a eficácia do filtro Anscombe/Wiener na redução do ruído Poisson o qual teve uma forte repercussão sobre os resultados da segmentação principalmente quando aplicado junto com a abordagem de segmentação Fuzzy que usa Pesos Dinâmicos. A relevância deste fato é o grande impacto no processo de quantificação dos volumes PET analisados.

\section{Agradecimentos}

Ao Laboratório de Engenharia Biomédica da Escola Politécnica da Universidade de São Paulo (LEB-EPUSP), ao Instituto do Coração do Hospital das Clínicas da Faculdade de Medicina da Universidade de São Paulo (InCor - HCFMUSP), ao Conselho Nacional de Desenvolvimento Científico e Tecnológico (CNPq), ao Programa de Estudante Convênio de Pós-Graduação (PEC-PG) e ao Ministério de Relações Exteriores Perú - Brasil.

\section{Referências}

Adams E, Asua J, Conde Olasagasti J, Erlichman M, Flynn K, Hurtado-Saracho I. Positron Emission Tomography:
Experience with PET and Synthesis of the Evidence. International Network of Agencies for Health Technology Assessment (INAHTA); 1999.

Anscombe FJ. The transformation of Poisson, binomial and negative binomial dat. Biometrika. 1948; 15:246-54.

Bethea RM, Duran RA. Statistical methods for engineers and scientists. New York: McHill Reckker; 1995.

Bushberg JT, Seibert JA, Leidholdt EM, Boone JM. The essencial physics of medical imaging. Lippincott Williams \& Wilkins Edition; 2002. Chapter 1: Introduction to Medical Imaging.p. 3-17.

Caetano R. Inovações e trajetórias tecnológicas no território das imagens médicas [tese]. Rio de Janeiro: Instituto de Medicina Social, Universidade do Estado do Rio de Janeiro; 2002.

De Almeida JP, Trindade MV, Gomes D, Fernandes CD, De Moraes W. O que é Medicina Nuclear? Portal dos profissionais de radiologia [Internet]. 2002. [acesso em 2010 set. 23]. Disponível em: http://www.rxinfo.com.br/

Dial R. Algorithm 360: Shortest path forest with topological ordering. Communications of the ACM. 1969; 12(11):632-3. http://dx.doi.org/10.1145/363269.363610

Dijkstra E. A note on two problems in conection with graphs. Numerische Mathematic. 1959; 1:269-271. http://dx.doi. org/10.1007/BF01386390

Fabey FH. State of the art in emission tomography equipment. Radiographics. 1996; 16(2):409-20. PMid:8966297.

Falcão AX, Stolfi J, Lotufo RA. The image foresting transform: theory, algorithms, and applications. IEEE Transactions Pattern Analysis and Machine Intelligence. 2004; 26(1):19-29. http://dx.doi.org/10.1109/TPAMI.2004.1261076

Fifer AM, Grossman W. Measurement of ventricular volumes, ejection fraction, mass, wall stress, and regional wall motion. Boston; 2000. Section V: Evaluation of Cardiac Function. Harvard Medical School; Coronary Care Unit, Massachusetts General Hospital.

Flórez Pacheco E. Quantificação da dinâmica de estruturas em imagens de Medicina Nuclear na modalidade PET [dissertação]. São Paulo: Escola Politécnica da Universidade de São Paulo; 2012. 200 p.

Gonzalez RC, Woods RE. Digital image processing. 3rd ed. New Jersey: Pearson Prentice Hall; 2008. Chap. 9: Morphological Image Processing.

Inouye T. Square Root Transform for the Analysis of Quantum Fluctuation in spectrum data. Nuclear Instruments and Methods. 1971; 91:581. http://dx.doi.org/10.1016/002 9-554X(71)90682-3

Johnson S, Bigelow C, Lee K, Pryor D, Jones R. Prediction of death and myocardial infarction by radionuclide angiocardiography in patients with suspected coronary artery disease. American Journal of Cardiology. 1991; 67:919-26. http://dx.doi.org/10.1016/0002-9149(91)90161-D 
Kass M, Witkin A, Terzopoulos D. Snakes: Active contour models. International Journal of Computer Vision. 1988; 321-31. http://dx.doi.org/10.1007/BF00133570

King, MA, Doherty PW, Schwinger RB. A Wiener filter for nuclear medicine images. Medical Physics. 1983; 10(6):876-80. PMid:6656698. doi: http:// dx.doi.org/10.1118/1.595352

Lage DM. Visualização de artérias coronárias epicárdicas em imagens ecocardiográficas tridimensionais com contraste de microbolhas [tese]. São Paulo: Universidade de São Paulo; 2010.

Lee JS. Digital image enhancement and noise filtering by use of local statistics. IEEE Transactions on Pattern Analysis and Machine Intelligence. 1980; PAMI-2:165-8. (Adaptive Wiener filter). http://dx.doi.org/10.1109/TPAMI.1980.4766994

Mascarenhas NDA, Santos CAN, Cruvinel PE. Transmition Tomography under Poisson noise using the Anscombe Transform and a Wiener Filter of the projections. Nuclear Instruments and Methods in Physics Research. 1999; 423:265-71. http://dx.doi.org/10.1016/ S0168-9002(98)00925-5

Marques Filho O, Vieira NH. Processamento digital de imagens. Rio de Janeiro: Editora Brasport; 1999.

Moraes MC, Furuie SS. Automatic coronary wall segmentation in intravascular ultrasound images using binary morphological reconstruction. Ultrasound in Medicine \& Biology. 2011; 37(9):1486-99. PMid:21741157. http:// dx.doi.org/10.1016/j.ultrasmedbio.2011.05.018

National Academy of Sciences. Mathematics and physics of emerging biomedical imaging. National Academy of Engineering/Institute of Medicine. Washington: National Academy Press; 1996. 233 p.

Nist/Sematech. Handbook of statistical methods [Internet]. 2011. [cited 2011 Nov 10] Available from: http://www.itl.nist.gov/div898/handbook/

Nyúl ALG, Falcão AX, Udupa JK. Fuzzy-connected 3D image segmentation at interactive speeds. Elsevier on Graphical Models. 2002; 64:259-81. http://dx.doi.org/10.1016/S10773169(02)00005-9

Oliveira R, Santos D, Ferreira D, Coelho P, Veiga F. Preparações radiofarmacêuticas e suas aplicações. Brazilian Journal of Pharmaceutical Sciences. 2006; 2(2):151-65.

Osher S, Fedkiw R. Level set methods and dynamic implicit surfaces. New York: Springer-Verlag; 2002.

Otsu N. Threshold selection method from gray-level histograms. IEEE Transactions on Systems, Man an Cybernet. 1979; 9(1):62-6. http://dx.doi.org/10.1109/ TSMC.1979.4310076

Pednekar AS, Kakadiaris IA. Image segmentation based on fuzzy connectedness using dynamic weights. IEEE Transactions on Image Processing. 2006; 15(6):1555-62. http://dx.doi.org/10.1109/TIP.2006.871165

Pedone MD, Castro I, Hatem D, Haertel JC, Feier F, Pandolfo F. Variações de parâmetros da função diastólica do ventrículo esquerdo de acordo com a idade através da ecocardiografia com Doppler Tissular. Arquivos Brasileiros de Cardiologia. 2004; 83(6):461-5. http://dx.doi.org/10.1590/ S0066-782X2004001800004

Peregrino AAF, Coelho CD, Vianna CMM, Coeli CM, Vianna D, Werneck GL, Magalhães REA, Caetano R. Avaliação tecnológica da tomografia de emissão de pósitrons (PET): revisão sistemática e proposta de estudo. Rio de Janeiro: CEPESC/IMS; 2004. Relatório 1. Síntese das Avaliações Tecnológicas sobre PET realizadas por Agências Internacionais de ATS na área de Oncologia.

Pertusa GJF. Técnicas de análisis de imágenes: Aplicaciones en biología. Editorial Maite Simon, Grupo Carduche; 2003. cap. 9. Procesado de Imagen, p. 141-61.

Robilotta CC. A tomografia por emissão de pósitrons: uma nova modalidade na medicina nuclear brasileira. Revista Panamericana de Salud Pública. 2006; 20(2-3):134-42. PMid:17199908. http://dx.doi.org/10.1590/ S1020-49892006000800010

Rodrigues I, Sanches J, Bioucas-Dias J. Denoising of medical images corrupted by poisson noise. In: ICIP 2008: Proceedings of the 15th IEEE International Conference Image Processing; 2008; San Diego. IEEE; 2008. p. 1756-9. http://dx.doi.org/10.1109/ICIP.2008.4712115

Romualdo LCS. Restauração de imagens mamográficas digitais utilizando o filtro de Wiener no domínio de Anscombe e o filtro inverso da MTF no domínio da frequência [dissertação]. São Paulo: Escola de Engenharia de São Carlos da Universidade de São Paulo; 2009. 189 p.

Russ JC. The image processing handbook. 3rd ed. North Carolina: IEEE Press; 1998. Chap. 9: 3D Image Acquisition.

Sarmento VA, Carvalho ACR, Luz IM. Evaluation of photon noise on digitalized images. Revista da Faculdade de Odontologia. 2004; 45(1):23-8.

Segars WP, Tsui BMW. MCAT TO XCAT: The evolution of 4-D computerized phantoms for imaging research. proceedings of the IEEE. 2009; 97(12):1954-68. http:// dx.doi.org/10.1109/JPROC.2009.2022417

Shaw L, Heinle S, Borges-Neto S, Kesler K, Coleman R, Jones R. Prognosis by measurements of left ventricular function during exercise. Journal of Nuclear Medicine. 1998; 39:140-46.

Souza S, De Oliveira A. Alguns comentários sobre a Teoria Fuzzy. Exata versão 1. Matemática Aplicada IME - USP; 1992; p. 139-47.

Tinois E. Imagem funcional - PET e fMRI. MultiCiência: Tecnologia para a Saúde. 2005; 5:1-14.

Udupa KJ, LaBlanc RV, Schmidt H. A methodology for evaluating image segmentation algortihms; 2002.

Udupa JK, Saha PK. Fuzzy connectedness and image segmentation. Procedings of IEEE. 2003; 91:1649-69. http://dx.doi.org/10.1109/JPROC.2003.817883

Udupa JK, Samarasekera S. Fuzzy connectedness and object definition: Theory, algorithms, and applications in image segmentation. Graphical Models Image Process. 1996; 58(3):246-61. http://dx.doi.org/10.1006/ gmip.1996.0021 
Wagner Junior HN. A brief history of positron emission tomography (PET). Seminars in Nuclear Medicine. 1998; 28(3):213-20. http://dx.doi.org/10.1016/ S0001-2998(98)80027-5

Whitehouse DJ. Handbook of surface metrology. England: Leicester; 1998.
Wikipedia. Ventrículo (coração) [Internet]. 2011. [acesso em 2011 jun 15] Disponível em: http://pt.wikipedia.org/ wiki/Ventr\%c3\%ADculo_card\%c3\%ADaco

Xu JP. Generalized gradient vector flow external forces for active contours. Signal Processing; 1998. http://dx.doi. org/10.1016/S0165-1684(98)00140-6

\section{Autores}

\section{Edward Flórez Pacheco*, Sérgio Shiguemi Furuie}

Laboratório de Engenharia Biomédica, Departamento de Engenharia de Telecomunicações e Controle, Escola Politécnica, Universidade de São Paulo - USP, Av. Professor Luciano Gualberto, Travessa 3, 158, CEP 05508-970, São Paulo, SP, Brasil. 